\title{
Extraintestinal pathogenic Escherichia coli: a combination of virulence with antibiotic resistance
}

\author{
Johann D. D. Pitout ${ }^{1,2,3}$ * \\ 1 Division of Microbiology, Calgary Laboratory Services, Calgary, AB, Canada \\ 2 Department of Pathology and Laboratory Medicine, University of Calgary, Calgary, AB, Canada \\ ${ }^{3}$ Microbiology and Infectious Diseases, University of Calgary, Calgary, AB, Canada
}

Edited by:

Stefania Stefani, University of

Catania, Italy

Reviewed by:

Neil Woodford, Health Protection

Agency, UK

Laura Pagani, University of Pavia, Italy

*Correspondence:

Johann D. D. Pitout, Division of

Microbiology, Calgary Laboratory

Services, No. 9, 3535 Research Road

Northwest, Calgary, AB, Canada T2L

$2 K 8$.

e-mail: johann.pitout@cls.ab.ca
Escherichia coli represents an incredible versatile and diverse enterobacterial species and can be subdivided into the following; (i) intestinal non-pathogenic, commensal isolates. (ii) Intestinal pathogenic isolates and (iii) extraintestinal pathogenic E. coli or ExPEC isolates. The presence to several putative virulence genes has been positively linked with the pathogenicity of ExPEC. E. coli remains one of the most frequent causes of nosocomial and community-acquired bacterial infections including urinary tract infections, enteric infections, and systemic infections in humans. ExPEC has emerged in 2000s as an important player in the resistance to antibiotics including the cephalosporins and fluoroquinolones. Most importantly among ExPEC is the increasing recognition of isolates producing "newer $\beta$-lactamases" that consists of plasmid-mediated AmpC $\beta$-lactamases (e.g., CMY), extended-spectrum $\beta$-lactamases (e.g., CTX-M), and carbapenemases (e.g., NDM). This review will highlight aspects of virulence associated with ExPEC, provide a brief overview of plasmid-mediated resistance to $\beta$-lactams including the characteristics of the successful international sequence types such as ST38, ST131, ST405, and ST648 among EXPEC.

Keywords: Escherichia coli, virulence, antimicrobial resistance

\section{INTRODUCTION}

Escherichia coli is an important cause of urinary tract infections (UTIs), enteric infections, and systemic infections in humans (Mandell et al., 2005). The systemic infections include bacteremia, nosocomial pneumonia, cholecystitis, cholangitis, peritonitis, cellulitis, osteomyelitis, and infectious arthritis. E. coli is also leading cause of neonatal meningitis.

Escherichia coli comprises of non-pathogenic commensal isolates that forms part of the normal flora of humans and various animals (Kaper et al., 2004). However, several variants have been described that cause infection of the gastrointestinal system (intestinal pathogenic E. coli) while others cause infections outside the gastrointestinal system (extraintestinal pathogenic E. coli or ExPEC). ExPEC incorporates the following variants; avian pathogenic E. coli, uropathogenic E. coli (UPEC), and those isolates responsible for septicemia and neonatal meningitis (Kaper et al., 2004).

Uropathogenic E. coli are the primary causes of communityacquired UTIs with an estimated $20 \%$ of women over the age of 18 years suffering from at least one UTI in their lifetime (Foxman, 2010). UPEC is responsible for 70-95\% of community-onset UTIs and approximately $50 \%$ of nosocomial UTIs, hence accounting for substantial morbidity, mortality, and medical expenses. Recurrent or relapsing UTIs are especially problematic in many individuals. The primary reservoir of UPEC is believed to be the human intestinal tract and employ diverse repertoire of virulence factors to colonize and infect the urinary tract in an ascending fashion (Foxman, 2010). However, community-onset clonal outbreaks of
UTIs, possibly due to the consumption of food contaminated with UPEC have also been described (Wiles et al., 2008). Additionally, there is some evidence that UPEC isolates can also be transmitted via sexual activities (Wiles et al., 2008).

The aim of this article is to highlight aspects of virulence associated with ExPEC, to provide a brief overview of the most important plasmid-mediated resistance to $\beta$-lactams including the characteristics of international sequence types among ExPEC.

\section{GENOMICS AND VIRULENCE FACTORS}

Virulence factors involve mechanisms that enable pathogenic bacteria to cause infections. Genomics offers an interesting tool for defining virulence factors; it can be used to identify genes encoding for specific factors that contributes to virulence in pathogens. However, the presence of a single factor rarely makes an organism virulent; a combination of factors will determine if a bacterium can cause infection (Dobrindt, 2005). Moreover, the determining factor is not simply the presence or absence of virulence-associated genes, but also their levels of expression, which can vary between pathogenic and non-pathogenic isolates.

The E. coli genome is composed of a conserved core of genes that provides the backbone of genetic information required for essential cellular processes and a flexible gene pool that harbors genetic information which provides properties that enables the bacterium to adapt to special environmental conditions (Dobrindt, 2005). The size of the flexible gene pool is determined by the acquisition and loss of genomic DNA. The parallel gain and loss of mobile genetic elements in the flexible portion of 
the gene pool such as bacteriophages, plasmids, genomic islands, transposons, insertion elements enabled the evolution of separate clones of E. coli (Dobrindt, 2005). A portion of the flexible gene pool consists of accessory genetic elements (including plasmids, transposons, insertion elements, prophages, and genomic islands), that can be either integrated into the chromosome or replicate independently as extrachromosomal elements. These elements can be laterally transferred to other species thus contributing to inter-, intra-species variability in genomic contents (Dobrindt, 2005).

The presence to several putative virulence genes has been positively linked with the pathogenicity of ExPEC. Phylogenetic analyses have shown that intestinal E. coli and ExPEC falls into four main phylogenetic groups namely A, B1, B2, and D (Herzer et al., 1990). ExPEC belongs mainly to group B2 and, to a lesser extent, to group $\mathrm{D}$ while intestinal commensal isolates tend to belong to groups A and B1. ExPEC isolates exhibit considerable genome diversity and possess a broad range of virulence-associated factors including toxins, adhesions, lipopolysaccharides, polysaccharide capsules, proteases, and invasins that are frequently encoded by pathogenic islands and other mobile DNA islands (Table 1). It seems that these putative virulence factors contribute to fitness (e.g., iron-uptake systems, bacteriocins, proteases, adhesins) of ExPEC and increase the adaptability, competitiveness, and ability to colonize the human body rather than being typical virulence factors directly involved in infection (Mokady et al., 2005).

Whether a commensal E. coli will develop into ExPEC requires the presence of functional genes directly contributing to pathogenesis and certain putative factors enabling the successful colonization of the host that enhances fitness and adaptation of the bacterium to its surroundings. This highlights the thin line between "virulence" and "fitness" and "colonization" factors in the definition of ExPEC virulence factors.

\section{ANTIMICROBIAL RESISTANCE IN ESCHERICHIA COLI}

The management of infections caused by ExPEC has been complicated by the emergence of antimicrobial resistance, especially since the late 1990s (Pitout and Laupland, 2008). The cephalosporins, fluoroquinolones, and trimethoprim-sulfamethoxazole are often used to treat community and hospital infections caused by $E$. coli and resistance to these agents is responsible for delays in appropriate therapy with subsequently increasing morbidity and mortality (Lautenbach et al., 2001; Tumbarello et al., 2007). Until the late 1990s ExPEC were relatively susceptible to first line antibiotics, however several surveillance studies during the 2000s across Europe, North and South America have shown that between 20 and $45 \%$ of ExPEC are resistant to first line antibiotics including the cephalosporins, fluoroquinolones, and trimethoprim-sulfamethoxazole (Foxman, 2010).

\section{RESISTANCE TO $\beta$-LACTAMS}

$\beta$-Lactam antibiotics, especially the third generation cephalosporins, are a major drug class used to treat serious community-onset or hospital-acquired infections caused by E. coli (Livermore and Woodford, 2006). Among E. coli, $\beta$-lactamase production remains the most important mediator of $\beta$-lactam resistance. $\beta$-lactamases are bacterial enzymes that inactivate $\beta$-lactam antibiotics by
Table 1 | Virulence-associated factors present in ExPEC.

\section{Virulence factor}

\begin{tabular}{|c|c|}
\hline \multicolumn{2}{|c|}{ ADHESINS } \\
\hline F10 papA & P fimbriae subunit variant \\
\hline papC & papACEFG, genes of $\mathrm{P}$ fimbriae operon \\
\hline papEFG & papACEFG, genes of $\mathrm{P}$ fimbriae operon \\
\hline$s f a / f o c$ & S or F1C fimbriae \\
\hline foc $G$ & F1C fimbriae adhesin \\
\hline iha & Adhesion siderophore \\
\hline $\mathrm{fimH}$ & Type 1 fimbriae \\
\hline tsh & temperature sensitive hemagglutinin \\
\hline hra & Heat-resistant agglutinin \\
\hline$a f a / d r a B C$ & Dr-binding adhesins \\
\hline \multicolumn{2}{|l|}{ TOXIN } \\
\hline hlyD & $\alpha$-Hemolysin \\
\hline sat & Secreted autotransporter toxin \\
\hline pic & Serine protease \\
\hline vat & Vacuolating toxin \\
\hline ast $A$ & Enteroaggregative E. coli toxin \\
\hline cnf1 & Cytotoxic necrotizing factor \\
\hline \multicolumn{2}{|c|}{ SIDEROPHORES } \\
\hline $\operatorname{iroN}$ & Salmochelin (siderophore) receptor \\
\hline fyuA & Yersiniabactin (siderophore) receptor \\
\hline ireA & Siderophore receptor \\
\hline iutA & Aerobactin (siderophore) receptor \\
\hline \multicolumn{2}{|l|}{ CAPSULE } \\
\hline kpsM II & kpsM II group 2 capsule \\
\hline K1 & K1 group 2 capsule variants \\
\hline K2 & K2 group 2 capsule variants \\
\hline K5 & K5 group 2 capsule variants \\
\hline kpsMT III & Group 3 capsule \\
\hline \multicolumn{2}{|c|}{ MISCELLANEOUS } \\
\hline usp & Uropathogenic-specific protein \\
\hline $\operatorname{traT}$ & Serum resistance-associated \\
\hline ompT & Outer membrane protease $T$ \\
\hline iss & Increased serum survival \\
\hline H7 flic & Flagellin variant \\
\hline $\operatorname{malX}$ & Pathogenicity island marker \\
\hline
\end{tabular}

hydrolysis, which results in ineffective compounds (Livermore and Woodford, 2006).

Most importantly is the increasing numbers of ExPEC isolates producing "newer $\beta$-lactamases" that includes the plasmidmediated AmpC $\beta$-lactamases (e.g., CMY types), extendedspectrum $\beta$-lactamases (ESBL; e.g., CTX-M types), and carbapenemases. The carbapenemases consists of three important classes; the class A (e.g., KPC types), the class B or metallo$\beta$-lactamases (MBLs), and class D or OXA types (Jacoby and Munoz-Price, 2005). CMY-2, CTX-Ms, and NDM types of $\beta$ lactamase are mostly responsible for the emerging resistance to the $\beta$-lactam antibiotics in E. coli. The characteristics of these newer $\beta$-lactamases including the KPC and OXA types are summarized in Table 2. Various carbapenemases including VIM, IPM, KPC, and OXA-48 $\beta$-lactamases have been described in members of the 
Table 2 | Newer $\beta$-lactamases in Escherichia coli.

\begin{tabular}{|c|c|c|c|c|}
\hline Enzymes & Classification & Examples & Spectrum of resistance & Inhibition \\
\hline \multirow[t]{3}{*}{ Extended-spectrum $\beta$-lactamases (ESBLs) } & Class A & CTX-M, TEM, SHV & Penicillins & Clavulanic acid \\
\hline & & & Cephalosporins & Tazobactam \\
\hline & & & Monobactams & Sulbactam \\
\hline \multirow[t]{4}{*}{ Plasmid-mediated AmpC $\beta$-lactamases } & Class C & CMY, FOX, ACT, MOX, ACC, DHA & Penicillins & Cloxacillin \\
\hline & & & Cephalosporins & \\
\hline & & & Monobactams & Boronic acid \\
\hline & & & Cephamycins & \\
\hline \multirow[t]{4}{*}{ Metallo- $\beta$-lactamases (MBLs) } & Class B & IMP, VIM, NDM & Penicillins & Metal chelators, \\
\hline & & & Cephalosporins & e.g., EDTA and \\
\hline & & & Cephamycins & dipicolinic acid \\
\hline & & & Carbapenems & \\
\hline \multirow[t]{4}{*}{ KPC carbapenemases } & Class A & $\mathrm{KPC}$ & Penicillins & Clavulanic acid \\
\hline & & & Cephalosporins & (weak) \\
\hline & & & Cephamycins & Tazobactam \\
\hline & & & Carbapenems & Boronic acid \\
\hline \multirow[t]{4}{*}{ OXA- $\beta$-lactamases } & Class D & OXA-48, -181 & Penicillins & Clavulanic acid \\
\hline & & & Temocillin & (weak) \\
\hline & & & $\beta$-lactamase combinations & $\mathrm{NaCl}$ \\
\hline & & & Carbapenems & \\
\hline
\end{tabular}

Enterobacteriaceae (including E. coli). However, these enzymes are less common among $E$. coli and will not be addressed in this review. It is therefore important to remember that in certain areas of the world, KPC and OXA-48-producing E. coli can be important causes of carbapenem resistance.

\section{EXTENDED-SPECTRUM $\beta$-LACTAMASES}

The most well known of the "newer" $\beta$-lactamases was first described in 1983 and have been named the ESBLs. These enzymes have the ability to hydrolyse the penicillins, cephalosporins, and monobactams, but not the cephamycins and carbapenems, and are inhibited by "classical" $\beta$-lactamase inhibitors such as clavulanic acid, sulbactam, and tazobactam (Paterson and Bonomo, 2005). Although ESBLs have been identified in a range of Enterobacteriaceae, they are most often present in E. coli and K. pneumoniae. The majority of ESBLs identified in clinical isolates during the 1980s1990s were the SHV or TEM types, which evolved from parent enzymes such as TEM-1, -2, and SHV-1 (Paterson and Bonomo, 2005). A different type of ESBL, named CTX-M $\beta$-lactamases, originated from environmental Kluyvera spp., and gained prominence in the 2000s with reports of clinical isolates of E. coli producing these enzymes from Europe, Africa, Asia, South and North America (Canton and Coque, 2006). Since the mid 2000s CTX$\mathrm{M} \beta$-lactamases had been identified in different members of the Enterobacteriaceae, but especially in E. coli, and have become the most widespread and common type of ESBL (Pitout and Laupland, 2008).

CTX-M-producing E. coli are important causes of communityonset UTIs, bacteremia and intra-abdominal infections (Canton and Coque, 2006). Risk factors associated with infections caused by CTX-M-producing E. coli include the following: repeat UTIs, underlying renal pathology, previous antibiotics including cephalosporins and fluoroquinolones, previous hospitalization, nursing home residents, older males and females, Diabetes Mellitus, underlying liver pathology, and international travel to high risk areas such as the Indian subcontinent (Rodriguez-Bano and Pascual, 2008). There are geographic variations in the prevalence of ExPEC that produce CTX-Ms. Surveys from several countries worldwide have illustrated an alarming trend of associated resistance to other classes of antimicrobial agents among CTX-Mproducing E. coli that included trimethoprim-sulfamethoxazole, tetracycline, gentamicin, tobramycin, and ciprofloxacin (Pitout and Laupland, 2008).

Currently, the most widespread and prevalent type of CTX-M enzyme among human clinical isolates of E. coli is CTX-M-15. ExPEC producing this enzyme often belong to the international uropathogenic sequence type named ST131 and to a lesser extend ST38, ST405, and ST648 (Peirano and Pitout, 2010). It seems that the intercontinental dissemination of these sequence types have in part contributed to the worldwide emergence of CTXM-15 producing $E$. coli. ST131 with CTX-M- $\beta$-lactamases when compared to other ESBL-producing E. coli was more likely to be resistant to antibiotics, to produce the aminoglycoside modifying enzyme $a a c(6)-I b-c r$, and cause community-acquired infections including urosepsis (Pitout et al., 2009). ST131 is not a single entity and can be subdivided into different clusters by other typing methods such as pulsed-field electrophoresis (Peirano et al., 2011a). It is therefore possible that variants of ST131 may differ in virulence profiles and ability to spread. The global distribution of ST131 cam also reflects repeated selection of local variants that have acquired certain resistance plasmids. A recent study from Canada showed that investigated the molecular epidemiology of ESBLs-producing E. coli causing bacteremia over an 11-year period (2000-2010) showed that ST131 was the most common 
and antimicrobial resistant sequence type and the influx of a single pulsotype of ST131 was responsible for a significant increase of ESBL-producing E. coli especially since 2007 (Peirano et al., 2011a).

Johnson et al. (2009) gave some insight into the origin of ST131 in North America. They studied 199 trimethoprimsulfamethoxazole-resistant and fluoroquinolone-resistant E. coli isolated from urines in Canada during 2002-2004 and identified ST131 in $23 \%$ of isolates and nearly all were fluoroquinoloneresistant (i.e., 99\%) but, notably, remained susceptible to the cephalosporins (i.e., only $2 \%$ of ST131 in that study were resistant to the cephalosporins; Johnson et al., 2009). Another study by Johnson et al. (2010) investigated the presence and virulence properties of ST131 among 127 ExPEC E. coli from the 2007 SENTRY and meropenem yearly susceptibility test information collection (MYSTIC) surveillance programs across the United States. Overall 54 (i.e., 17\%) belonged to ST131, but interestingly this sequence type included $52 \%$ of isolates that showed resistance to $\geq 3$ antimicrobial classes. ST131 has a significant higher virulence score that other ExPEC and certain virulence factors such as uropathogenicspecific protein ( $u s p)$; outer membrane protein (ompT); secreted autotransporter toxin (sat), aerobactin receptor ( $i u t A$ ), and pathogenicity island marker ( $\operatorname{malX}$ ) were associated with this sequence type. Their results showed that ST131 had distinctive virulence and resistance profiles and concluded that the combination of antimicrobial resistance and virulence may be responsible for the epidemiological success of this sequence type.

Studies have recently identified different sequence types such as ST38, ST405, and ST648 among CTX-M-producing E. coli. ST38 with CTX-M-9, -14, and -15 has previously been described in clinical isolates from Japan (Suzuki et al., 2009), the Netherlands (van der Bij et al., 2011), Korea (Kim et al., 2011), and Tanzania (Mshana et al., 2011). This sequence type is also associated with OXA-48 (Poirel et al., 2011) and NDM-1 (Yamamoto et al., 2011). ST405 with various types of CTX-Ms has a worldwide distribution (Coque et al., 2008; Jones et al., 2008; Mihaila et al., 2010; Smet et al., 2010) while ST648 with CTX-M-15, -32 are present in poultry from Spain (Cortes et al., 2010) and humans in China (Zong and Yu, 2010) and the Netherlands (van der Bij et al., 2011). ST405 and ST648 are also associated with NDM $\beta$-lactamases (Hornsey et al., 2011; Mushtaq et al., 2011). These reports suggest that they might also play a role in the worldwide distribution and success of CTX-M-producing E. coli.

It is evident today that CTX-M-producing E. coli is a major player in the world of antimicrobial resistance. A report from the Infectious Diseases Society of America listed ESBL-producing E. coli as a priority drug-resistant microbe to which new therapies are urgently needed (Talbot et al., 2006).

\section{PLASMID-MEDIATED AmpC $\beta$-LACTAMASES}

Escherichia coli possess a chromosomal gene that encodes for an AmpC $\beta$-lactamase. Usually, low amounts of this $\beta$-lactamases are produced because the AmpC gene is regulated by a weak promoter and a strong attenuator (Olsson et al., 1982). Occasionally, cephamycin-resistant strains produce plasmid-mediated $\beta$-lactamases derived from bacteria with chromosomally encoded, AmpC-cephalosporinases such as Enterobacter, Citrobacter feundii,
Morganella morganii, Aeromonas spp., and Hafnia alvei (Philippon et al., 2002). These $\beta$-lactamases at high levels, hydrolyse penicillins, most cephalosporins, cephamycins, and monobactams, but not the fourth generation cephalosporins (e.g., cefepime) and carbapenems. AmpC enzymes are not inhibited by "classical" $\beta$-lactamase inhibitors such as clavulanic acid, sulbactam, and tazobactam although boronic acids and cloxacillin have shown to be good inhibitors (Doi and Paterson, 2007).

Just like ESBL-producing bacteria, organisms with plasmidmediated AmpC enzymes have mostly been responsible for nosocomial outbreaks on a worldwide basis during the late 1980s and 1990s, although the risk factors associated with infection are not as well defined as those associated with ESBL-producing bacteria (Philippon et al., 2002). In a study reported from Korea, patients infected by plasmid-mediated AmpC-producing organisms had similar clinical features and outcomes to those patients infected with TEM- or SHV-related ESBL producers (Pai et al., 2004).

The SENTRY Antimicrobial Surveillance Program in the USA found plasmid-mediated AmpC $\beta$-lactamases in 2\% of 1429 E. coli isolates from 30 centers; with CMY-2, FOX-5, and DHA-1 being identified (Deshpande et al., 2006). A UK study found acquired cephamycinases in $49 \%$ of cefoxitin-resistant E. coli (Woodford et al., 2007).

It seems that CMY-2, derived ancestrally from Citrobacter spp., is the most common plasmid-mediated cephamycinase reported in E. coli from different areas of the world. Recent hospital surveys from Asia, North America, and Europe have shown that the CMY types are present in E. coli from Asia, North America, and Europe (Alvarez et al., 2004; Mulvey et al., 2005; Moland et al., 2006; Woodford et al., 2007; Li et al., 2008). A population-based study conducted in Calgary, concluded that in this large Canadian region, CMY-2-producing $E$. coli is an emerging pathogen in the community that commonly causes UTIs in older women (Pitout et al., 2007). This was followed by two reports from Washington and Nebraska which showed Enterobacteriaceae producing CMY, ACC, and DHA types of AmpC $\beta$-lactamases are present in clinical isolates from outpatient clinics in the USA (Hanson et al., 2008; Qin et al., 2008).

\section{METALLO- $\beta$-LACTAMASES (NDM-1)}

Recently, a new type of MBL, named NDM, was described in $K$. pneumoniae and E. coli recovered from a Swedish patient who was hospitalized in New Delhi, India (Yong et al., 2009). MBLs have the ability to hydrolyse a wide variety of $\beta$-lactams, including the penicillins, cephalosporins, and carbapenems, but not the monobactams (i.e., aztreonam), and are inhibited by metal chelators such as EDTA (Table 2). The majority of NDM-1-producing bacteria are broadly resistant to various drug classes and also carry a diversity of other resistance mechanisms (e.g., to aminoglycosides and fluoroquinolones), which leaves limited treatment options (Nordmann et al., 2011a).

Kumarasamy et al. (2010), provide compelling evidence that NDM-producing Enterobacteriaceae (mostly K. pneumoniae and E. coli) are widespread in India and Pakistan. They also found that many UK patients infected with NDM-producing bacteria had recently traveled to India to undergo several types of medical procedures. The patients presented with a variety of hospital- 
and community-associated infections with UTIs being the most common clinical syndrome. Recent reports from the subcontinent (including India, Pakistan, and Bangladesh) show that the distribution of NDM $\beta$-lactamases among Enterobacteriaceae are widespread through these countries (Castanheira et al., 2011a,b; Lascols et al., 2011): e.g., a hospital in Varanasi in Northern India identified NDM-1 prevalence rate of $6 \%$ among E. coli $(n=528)$ from outpatients and hospitalized patients between February 2010 and July 2010 (Seema et al., 2011), 7\% among E. coli from a major hospital in Mumbai, India (Deshpande et al., 2010), whereas 15\% (30/200) of in- and outpatients in Rawalpindi, Pakistan carried E. coli with NDM-1 in their gut flora (Perry et al., 2011).

Since mid-August 2010, NDM-1-positive bacteria have been reported worldwide, except in Central and South America (Nordmann et al., 2011b). Most are Enterobacteriaceae from patients hospitalized in 2009 and 2010 with an epidemiological link to the Indian subcontinent. Recent findings suggest that the Balkan states and the Middle East might act as secondary reservoirs for the spread of NDM-1, which may or may not initially have reached these countries from the Indian subcontinent. Enterobacteriaceae with NDM-1 have been recovered from many clinical settings, reflecting the disease spectra of these opportunistic bacteria, including hospital and community-onset UTIs, septicemia, pulmonary infections, peritonitis, device-associated infections, and soft tissue infections (Castanheira et al., 2011a; Nordmann et al., 2011a,b). NDM-1-positive bacteria have been recovered from the gut flora of travelers returning from the Indian subcontinent and undergoing microbiological investigation for unrelated diarrheal symptoms (Leverstein-Van Hall et al., 2010). There is also widespread environmental contamination by NDM-1-positive bacteria in New Delhi (Walsh et al., 2011).

There is no evidence that $E$. coli that produce NDM are more virulent than other isolates, however recent studies described presence of NDM $\beta$-lactamases in the very successful $E$. coli sequence type ST131 with an identical virulence genotype than ST131 that produce CTX-M $\beta$-lactamases (Peirano et al., 2011b). Of interest,

\section{REFERENCES}

Alvarez, M., Tran, J. H., Chow, N., and Jacoby, G. A. (2004). Epidemiology of conjugative plasmidmediated AmpC beta-lactamases in the United States. Antimicrob. Agents Chemother. 48, 533-537.

Canton, R., and Coque, T. M. (2006). The CTX-M beta-lactamase pandemic. Curr. Opin. Microbiol. 9, 466-475.

Castanheira, M., Deshpande, L. M., Mathai, D., Bell, J. M., Jones, R. N., and Mendes, R. E. (2011a). Early dissemination of NDM-1 - and OXA-181-producing Enterobacteriaceae in Indian hospitals: report from the SENTRY Antimicrobial Surveillance Program, 2006-2007. Antimicrob. Agents Chemother. 55, 1274-1278.

Castanheira, M., Mendes, R. E., Woosley, L. N., and Jones, R. N.

ST131 with VIM and KPC carbapenemases have also recently been described (Mantengoli et al., 2011; Morris et al., 2011). Due to the very resistant nature of these NDM-producing E. coli, the treatment of infections due to these bacteria will remain a challenge to physicians. Antibiotics such as colistin, tigecycline, and fosfomycin show the best activity against NDM-producing bacteria (Nordmann et al., 2011b). However, Stone et al. (2011) recently described a breakthrough bacteremia caused by E. coli that produce NDM while the patient was treated with tigecycline; the isolate developed resistance to tigecycline.

\section{SUMMARY}

Escherichia coli is an important cause of UTIs, enteric infections, and systemic infections in humans. The presence to several putative virulence genes has been positively linked with the pathogenicity of ExPEC including toxins, adhesions, lipopolysaccharides, polysaccharide capsules, proteases, and invasins that are frequently encoded by pathogenic islands and other mobile DNA islands.

Up to the late 1990s ExPEC was relatively susceptible to first line antibiotics, however several surveillance studies during the 2000s across the world have shown increasing resistance to first line antibiotics including the cephalosporins, fluoroquinolones, and trimethoprim-sulfamethoxazole. The "newer $\beta$-lactamases" that consists of plasmid-mediated AmpC $\beta$-lactamases (e.g., CMY types), ESBL (e.g., CTX-M types), and carbapenemases (e.g., NDM) are important causes of resistance to $\beta$-lactam antibiotics among ExPEC.

Recent reports suggest that certain sequence types such as ST38, ST131, ST405, and ST648 might play an important role in the worldwide distribution and success of CTX-M-producing E. coli. Future investigations should be undertaken to study the microbiological and ecological factors that make CTX-M and NDM-producing E. coli such successful pathogens including welldesigned epidemiological, clinical, and molecular studies to understand the dynamics of transmission, risk factors, and reservoirs for ST131.

coli strains from chicken and pig farms in Spain. Appl. Environ. Microbiol. 76, 2799-2805.

Deshpande, L. M., Jones, R. N., Fritsche, T. R., and Sader, H. S. (2006). Occurrence of plasmidic AmpC type beta-lactamase-mediated resistance in Escherichia coli: report from the SENTRY Antimicrobial Surveillance Program (North America, 2004). Int. J. Antimicrob. Agents 28, 578-581.

Deshpande, P., Rodrigues, C., Shetty, A., Kapadia, F., Hedge, A., and Soman, R. (2010). New Delhi metallo-beta lactamase (NDM-1) in Enterobacteriaceae: treatment options with carbapenems compromised. J. Assoc. Physicians India 58, 147-149.

Dobrindt, U. (2005). (Patho-)Genomics of Escherichia coli. Int. J. Med. Microbiol. 295, 357-371.

Doi, Y., and Paterson, D. L. (2007). Detection of plasmid-mediated class
C beta-lactamases. Int. J. Infect. Dis. 11, 191-197.

Foxman, B. (2010). The epidemiology of urinary tract infection. Nat. Rev. Urol. 7, 653-660.

Hanson, N. D., Moland, E. S., Hong, S. G., Propst, K., Novak, D. J. and Cavalieri, S. J. (2008). Surveillance of community-based reservoirs reveals the presence of CTX$M$, imported AmpC, and OXA30 beta-lactamases in urine isolates of Klebsiella pneumoniae and Escherichia coli in a U.S. community. Antimicrob. Agents Chemother. 52, 3814-3816.

Herzer, P. J., Inouye, S., Inouye, M., and Whittam, T. S. (1990). Phylogenetic distribution of branched RNAlinked multicopy single-stranded DNA among natural isolates of Escherichia coli. J. Bacteriol. 172, 6175-6181. 
Hornsey, M., Phee, L., and Wareham, D.W. (2011). A novel variant, NDM-5, of the New Delhi metallobeta-lactamase in a multidrugresistant Escherichia coli ST648 isolate recovered from a patient in the United Kingdom. Antimicrob. Agents Chemother. 55, 5952-5954.

Jacoby, G. A., and Munoz-Price, L. S. (2005). The new beta-lactamases. $N$. Engl. J. Med. 352, 380-391.

Johnson, J. R., Johnston, B., Clabots, C., Kuskowski, M. A., and Castanheira, M. (2010). Escherichia coli sequence type ST131 as the major cause of serious multidrug-resistant E. coli infections in the United States. Clin. Infect. Dis. 51, 286-294.

Johnson, J. R., Menard, M., Johnston, B., Kuskowski, M. A., Nichol, K., and Zhanel, G. G. (2009). Epidemic clonal groups of Escherichia coli as a cause of antimicrobial-resistant urinary tract infections in Canada, 2002 to 2004. Antimicrob. Agents Chemother. 53, 2733-2739.

Jones, G. L., Warren, R. E., Skidmore, S. J., Davies, V. A., Gibreel, T., and Upton, M. (2008). Prevalence and distribution of plasmid-mediated quinolone resistance genes in clinical isolates of Escherichia coli lacking extended-spectrum beta-lactamases. J. Antimicrob. Chemother. 62, 1245-1251.

Kumarasamy, K. K., Toleman, M. A., Walsh, T. R., Bagaria, J., Butt, F., Balakrishnan R. Chaudhary, U., Doumith, M., Giske, C. G., Irfan, S., Krishnan, P., Kumar, A. V., Maharjan, S., Mushtaq, S., Noorie, T., Paterson, D. L., Pearson, A., Perry, C., Pike, R., Rao, B., Ray, U., Sarma, J. B., Sharma, M., Sheridan, E., Thirunarayan, M. A., Turton, J., Upadhyay, S., Warner, M., Welfare, W., Livermore, D. M., and Woodford, N. (2010). Emergence of a new antibiotic resistance mechanism in India, Pakistan, and the UK: a molecular, biological, and epidemiological study. Lancet Infect. Dis. 10, 597-602.

Kaper, J. B., Nataro, J. P., and Mobley, H. L. (2004). Pathogenic Escherichia coli. Nat. Rev. Microbiol. 2, 123-140.

Kim, J., Bae, I. K., Jeong, S. H., Chang, C. L., Lee, C. H., and Lee, K. (2011). Characterization of IncF plasmids carrying the blaCTX-M-14 gene in clinical isolates of Escherichia coli from Korea. J. Antimicrob. Chemother. 66, 1263-1268.

Lascols, C., Hackel, M., Marshall, S. H., Hujer, A. M., Bouchillon, S., Badal, R., Hoban, D., and Bonomo, R. A. (2011). Increasing prevalence and dissemination of NDM-1 metallobeta-lactamase in India: data from the SMART study (2009). J. Antimicrob. Chemother. 66, 1992-1997.

Lautenbach, E., Patel, J. B., Bilker, W. B., Edelstein, P. H., and Fishman, N. O. (2001). Extended-spectrum betalactamase-producing Escherichia coli and Klebsiella pneumoniae: risk factors for infection and impact of resistance on outcomes. Clin. Infect. Dis. 32, 1162-1171.

Leverstein-Van Hall, M. A., Stuart, J. C., Voets, G. M., Versteeg, D., Tersmette, T., and Fluit, A. C. (2010). Global spread of New Delhi metallo-betalactamase 1. Lancet Infect. Dis. 10, 830-831.

Li, Y., Li, Q., Du, Y., Jiang, X., Tang, J., Wang, J., Li, G., and Jiang, Y. (2008). Prevalence of plasmidmediated AmpC beta-lactamases in a Chinese university hospital from 2003 to 2005: first report of CMY2-Type AmpC beta-lactamase resistance in China. J. Clin. Microbiol. 46, 1317-1321.

Livermore, D. M., and Woodford, N. (2006). The beta-lactamase threat in Enterobacteriaceae, Pseudomonas and Acinetobacter. Trends Microbiol. 14, 413-420.

Mandell, G. L., Douglas, R. G., Bennett, J. E., and Dolin, R. (2005). Mandell, Douglas, and Bennett's Principles and Practice of Infectious Diseases, 6th Edn. New York: Churchill Livingstone.

Mantengoli, E., Luzzaro, F., Pecile, P., Cecconi, D., Cavallo, A., Attala, L., Bartoloni, A., and Rossolini, G. M. (2011). Escherichia coli ST131 producing extended-spectrum betalactamases plus VIM-1 carbapenemase: further narrowing of treatment options. Clin. Infect. Dis. 52, 690-691.

Mihaila, L., Wyplosz, B., Clermont, O., Garry, L., Hipeaux, M. C., Vittecoq, D., Dussaix, E., Denamur, E., and Branger, C. (2010). Probable intrafamily transmission of a highly virulent CTX-M-3producing Escherichia coli belonging to the emerging phylogenetic subgroup D2 O102-ST405 clone. J. Antimicrob. Chemother. 65, 1537-1539.

Mokady, D., Gophna, U., and Ron, E. Z. (2005). Virulence factors of septicemic Escherichia coli strains. Int. J. Med. Microbiol. 295, 455-462.

Moland, E. S., Hanson, N. D., Black, J. A., Hossain, A., Song, W., and Thomson, K. S. (2006). Prevalence of newer beta-lactamases in gramnegative clinical isolates collected in the United States from 2001 to 2002. J. Clin. Microbiol. 44, 3318-3324.
Morris, D., Boyle, F., Ludden, C., Condon, I., Hale, J., O'Connell, N., Power, L., Boo, T. W., Dhanji, H., Lavallee, C., Woodford, N., and Cormican, M. (2011). Production of KPC-2 carbapenemase by an Escherichia coli clinical isolate belonging to the international ST131 clone. Antimicrob. Agents Chemother. 55, 4935-4936.

Mshana, S. E., Imirzalioglu, C., Hain, T., Domann, E., Lyamuya, E. F., and Chakraborty, T. (2011). Multiple ST clonal complexes, with a predominance of ST131, of Escherichia coli harbouring blaCTXM-15 in a tertiary hospital in Tanzania. Clin. Microbiol. Infect. 17, 1279-1282.

Mulvey, M. R., Bryce, E., Boyd, D. A., Ofner-Agostini, M., Land, A. M., Simor, A. E., and Paton, S. (2005). Molecular characterization of cefoxitin-resistant Escherichia col from Canadian hospitals. Antimicrob. Agents Chemother. 49, 358-365.

Mushtaq, S., Irfan, S., Sarma, J. B. Doumith, M., Pike, R., Pitout, J., Livermore, D. M., and Woodford, N. (2011). Phylogenetic diversity of Escherichia coli strains producing NDM-type carbapenemases. J. Antimicrob. Chemother. 66, 2002-2005.

Nordmann, P., Naas, T., and Poirel, L. (2011a). Global spread of carbapenemase-producing Enterobacteriaceae. Emerg. Infect. Dis. 17, 1791-1798.

Nordmann, P., Poirel, L., Walsh, T. R. and Livermore, D. M. (2011b). The emerging NDM carbapenemases. Trends Microbiol. 19, 588-595.

Olsson, O., Bergstrom, S., and Normark, S. (1982). Identification of a novel ampC beta-lactamase promoter in a clinical isolate of Escherichia coli. EMBO J. 1, 1411-1416.

Pai, H., Kang, C. I., Byeon, J. H., Lee, K. D., Park, W. B., Kim, H. B., Kim, E. C., Oh, M. D., and Choe, K. W. (2004). Epidemiology and clinical features of bloodstream infections caused by AmpC-typebeta-lactamase-producing Klebsiella pneumoniae. Antimicrob. Agents Chemother. 48, 3720-3728.

Paterson, D. L., and Bonomo, R. A. (2005). Extended-spectrum betalactamases: a clinical update. Clin. Microbiol. Rev. 18, 657-686.

Peirano, G., and Pitout, J. D. (2010). Molecular epidemiology of Escherichia coli producing CTX-M beta-lactamases: the worldwide emergence of clone ST131 O25:H4. Int. J. Antimicrob. Agents 35, 316-321.
Peirano, G., van der Bij, A. K., Gregson, D. B., and Pitout, J. D. (2011a). Molecular epidemiology over an eleven-year period (2000-10) of extended-spectrum beta-lactamaseproducing Escherichia coli causing bacteraemia in a centralized Canadian region. J. Clin. Microbiol. doi: 10.1128/JCM.06025-11. [Epub ahead of print].

Peirano, G., Schreckenberger, P. C., and Pitout, J. D. (2011b). Characteristics of NDM-1-producing Escherichia coli isolates that belong to the successful and virulent clone ST131. Antimicrob. Agents Chemother. 55, 2986-2988.

Perry, J. D., Naqvi, S. H., Mirza, I. A., Alizai, S. A., Hussain, A., Ghirardi, S., Orenga, S., Wilkinson, K., Woodford, N., Zhang, J., Livermore, D. M., Abbasi, S. A., and Raza, M. W. (2011). Prevalence of faecal carriage of Enterobacteriaceae with NDM-1 carbapenemase at military hospitals in Pakistan, and evaluation of two chromogenic media. J. Antimicrob. Chemother. 66, 2288-2294.

Philippon, A., Arlet, G., and Jacoby, G. A. (2002). Plasmid-determined AmpCtype beta-lactamases. Antimicrob. Agents Chemother. 46, 1-11.

Pitout, J. D., Gregson, D. B., Campbell, L., and Laupland, K. B. (2009). Molecular characteristics of extended-spectrum-betalactamase-producing Escherichia coli isolates causing bacteremia in the Calgary Health Region from 2000 to 2007: emergence of clone ST131 as a cause of communityacquired infections. Antimicrob. Agents Chemother. 53, 2846-2851.

Pitout, J. D., Gregson, D. B., Church, D. L., and Laupland, K. B. (2007). Population-based laboratory surveillance for AmpC betalactamase-producing Escherichia coli, Calgary. Emerg. Infect. Dis. 13, 443-448.

Pitout, J. D., and Laupland, K. B. (2008). Extended-spectrum betalactamase-producing Enterobacteriaceae: an emerging public-health concern. Lancet. Infect. Dis. 8, 159-166.

Poirel, L., Bernabeu, S., Fortineau, N., Podglajen, I., Lawrence, C., and Nordmann, P. (2011) Emergence of OXA-48-producing Escherichia coli clone ST38 in France. Antimicrob. Agents Chemother. 55, 4937-4938.

Qin, X., Zerr, D. M., Weissman, S. J., Englund, J. A., Denno, D. M., Klein, E. J., Tarr, P. I., Kwong, J., Stapp, J. R., Tulloch, L. G., and Galanakis, 
E. (2008). Prevalence and mechanisms of broad-spectrum betalactam resistance in Enterobacteriaceae: a children's hospital experience. Antimicrob. Agents Chemother. 52, 3909-3914.

Rodriguez-Bano, J., and Pascual, A. (2008). Clinical significance of extended-spectrum betalactamases. Expert Rev. Anti. Infect. Ther. 6, 671-683.

Seema, K., Ranjan Sen, M., Upadhyay, S., and Bhattacharjee, A. (2011). Dissemination of the New Delhi metallo-beta-lactamase-1 (NDM-1) among Enterobacteriaceae in a tertiary referral hospital in north India. J. Antimicrob. Chemother. 66, 1646-1647.

Smet, A., Martel, A., Persoons, D., Dewulf, J., Heyndrickx, M., Claeys, G., Lontie, M., Van Meensel, B., Herman, L., Haesebrouck, F., and Butaye, P. (2010). Characterization of extended-spectrum betalactamases produced by Escherichia coli isolated from hospitalized and nonhospitalized patients: emergence of CTX-M-15-producing strains causing urinary tract infections. Microb. Drug Resist. 16, 129-134.

Stone, N. R., Woodford, N., Livermore, D. M., Howard, J., Pike, R., Mushtaq, S., Perry, C., and Hopkins, S. (2011). Breakthrough bacteraemia due to tigecycline-resistant Escherichia coli with New Delhi metallo-beta-lactamase (NDM)-1 successfully treated with colistin in a patient with calciphylaxis. J. Antimicrob. Chemother. 66, 2677-2678.

Suzuki, S., Shibata, N., Yamane, K., Wachino, J., Ito, K., and Arakawa, Y. (2009). Change in the prevalence of extended-spectrum-betalactamase-producing Escherichia coli in Japan by clonal spread. J. Antimicrob. Chemother. 63, 72-79.

Talbot, G. H., Bradley, J., Edwards, J. E. Jr., Gilbert, D., Scheld, M., and Bartlett, J. G. (2006). Bad bugs need drugs: an update on the development pipeline from the Antimicrobial Availability Task Force of the Infectious Diseases Society of America. Clin. Infect. Dis. 42, 657-668.

Tumbarello, M., Sanguinetti, M., Montuori, E., Trecarichi, E. M., Posteraro, B., Fiori, B., Citton, R., D’Inzeo, T., Fadda, G., Cauda, R., and Spanu, T. (2007). Predictors of mortality in patients with bloodstream infections caused by extended-spectrumbeta-lactamase-producing Enterobacteriaceae: importance of inadequate initial antimicrobial treatment. Antimicrob. Agents Chemother. 51, 1987-1994.

van der Bij, A. K., Peirano, G. Goessens, W. H., van der Vorm, E. R., van Westreenen, M., and Pitout, J. D. (2011). Clinical and molecular characteristics of extended-spectrum-betalactamase-producing Escherichia coli causing bacteremia in the Rotterdam area, Netherlands. Antimicrob. Agents Chemother. 55, 3576-3578.

Walsh, T. R., Weeks, J., Livermore, D. M. and Toleman, M. A. (2011). Dissemination of NDM-1 positive bacteria in the New Delhi environment and its implications for human health: an environmental point prevalence study. Lancet Infect. Dis. 11, 355-362.

Wiles, T. J., Kulesus, R. R., and Mulvey, M. A. (2008). Origins and virulence mechanisms of uropathogenic Escherichia coli. Exp. Mol. Pathol. 85 11-19.

Woodford, N., Reddy, S., Fagan, E. J., Hill, R. L., Hopkins, K. L., Kaufmann, M. E., Kistler, J., Palepou, M. F., Pike, R., Ward, M. E., Cheesbrough, J., and Livermore, D. M. (2007). Wide geographic spread of diverse acquired AmpC betalactamases among Escherichia coli and Klebsiella spp. in the UK and Ireland. J. Antimicrob. Chemother. 59, 102-105.

Yamamoto, T., Takano, T., Iwao, Y. and Hishinuma, A. (2011). Emergence of NDM-1-positive capsulated Escherichia coli with high resistance to serum killing in Japan. J. Infect. Chemother. 17, 435-439.

Yong, D., Toleman, M. A., Giske, C. G., Cho, H. S., Sundman, K., Lee, K., and Walsh, T. R. (2009). Characterization of a new metallo-betalactamase gene, bla(NDM-1), and a novel erythromycin esterase gene carried on a unique genetic structure in Klebsiella pneumoniae sequence type 14 from India. Antimicrob. Agents Chemother. 53, 5046-5054

Zong, Z., and Yu, R. (2010). Escherichia coli carrying the blaCTX-M-15 gene of ST648. J. Med. Microbiol. 59(Pt 12), 1536-1537.

Conflict of Interest Statement: The author declares that the research was conducted in the absence of any commercial or financial relationships that could be construed as a potential conflict of interest.

Received: 12 December 2011; paper pending published: 03 January 2012; accepted: 05 January 2012; published online: 19 January 2012.

Citation: Pitout JDD (2012) Extraintestinal pathogenic Escherichia coli: a combination of virulence with antibiotic resistance. Front. Microbio. 3:9. doi 10.3389/fmicb.2012.00009

This article was submitted to Frontiers in Antimicrobials, Resistance and Chemotherapy, a specialty of Frontiers in Microbiology.

Copyright (c) 2012 Pitout. This is an open-access article distributed under the terms of the Creative Commons Attribution Non Commercial License, which permits non-commercial use, distribution, and reproduction in other forums, provided the original authors and source are credited. 